\title{
No Deterioration in Clinical Outcomes of Carbon Ion Radiotherapy for Sarcopenia Patients with Hepatocellular Carcinoma
}

\author{
SHINTARO SHIBA ${ }^{1}$, KEI SHIBUYA ${ }^{1}$, HIROYUKI KATOH ${ }^{2}$, YOSHINORI KOYAMA ${ }^{3}$, \\ MASAHIKO OKAMOTO ${ }^{1}$, TAKANORI ABE ${ }^{1}$, TATSUYA OHNO ${ }^{2}$ and TAKASHI NAKANO ${ }^{1}$ \\ ${ }^{1}$ Department of Radiation Oncology, Gunma University Graduate School of Medicine, Gunma, Japan; \\ ${ }^{2}$ Gunma University Heavy Ion Medical Center, Gunma, Japan; \\ ${ }^{3}$ Department of Diagnostic Radiology, Shibukawa Medical Center, Gunma, Japan
}

\begin{abstract}
Background/Aim: The relationship between sarcopenia and prognosis in carbon ion radiotherapy (C-ion $R T)$ for hepatocellular carcinoma (HCC) has not yet been reported, therefore we analyzed the presence or absence of sarcopenia before C-ion RT as a prognostic factor for patients with HCC. Patients and Methods: Data were retrospectively collected for patients who had undergone C-ion RT for HCC between September 2010 and December 2016. For defining the presence or absence of sarcopenia, skeletal muscles in the third lumbar vertebrae level were measured. Clinical outcomes were compared in the sarcopenia and non-sarcopenia groups. Results: Of the 68 patients who were analyzed, 22 were classified into the sarcopenia and 46 into the non-sarcopenia groups. Median follow-up of patients was 33.5 months. The three-year overall survival (OS) rates in the sarcopenia and non-sarcopenia groups were $66 \%$ and $77 \%$, respectively $(p=0.51)$. Conclusion: Sarcopenia was not a prognostic factor for patients with HCC treated with $C$-ion $R T$, which was effective in HCC patients with sarcopenia without worsening the OS.
\end{abstract}

The number of elderly patients with age-related loss of skeletal muscle mass (primary sarcopenia) has been on the rise in recent times. In addition, patients with cancer suffer from various metabolic disorders and malnutrition that may cause secondary sarcopenia, which is characterized by a loss

This article is freely accessible online.

Correspondence to: Shintaro Shiba, Department of Radiation Oncology, Gunma University Graduate School of Medicine, 3-3922 Syowa-machi, Maebashi, Gunma 371-8511, Japan. Tel: +81 272208383, Fax: +81 272208397, e-mail: shiba4885@yahoo.co.jp

Key Words: Carbon ion radiotherapy, hepatocellular carcinoma, sarcopenia. of skeletal muscle mass in an early stage, unlike malnutrition which is caused by starvation. Several researchers reported that preoperative skeletal muscle mass is associated with the prognosis of cancers (1-4).

Bioelectrical impedance method is frequently used for assessing skeletal muscle mass. Recently, computed tomography (CT) and magnetic resonance imaging (MRI) have been well established for the measurement of skeletal muscle mass at the third lumbar vertebrae level because of objectivity and accuracy (5).

Hepatocellular carcinoma (HCC) is the sixth-most frequently occurring cancer type and the third major cause of cancer-related death worldwide (6). Numerous local treatment options can be employed for treating HCC, with particle therapy, such as proton beam therapy and carbon ion radiotherapy (C-ion RT), being one of the less-invasive options $(7,8)$. Most patients with HCC have a history of chronic liver disease resulting from alcohol abuse, or infection with hepatitis C or B virus, so that patients with HCC already are in a state of secondary sarcopenia caused by cirrhosis or chronic hepatitis. Harimoto et al. reported the clinical outcomes of surgery and noted a remarkable difference in overall survival (OS) because of the difference in preoperative skeletal muscle mass (2). However, the relationship between sarcopenia and prognosis in particle therapy for HCC has not yet been reported. In the present study, a retrospective analysis was conducted on the presence or absence of sarcopenia before $\mathrm{C}$-ion $\mathrm{RT}$ as a prognostic factor for HCC.

\section{Patients and Methods}

Patients. This retrospective analysis was performed using the medical records of patients treated with C-ion RT for HCC at our hospital between September 2010 and December 2016. The diagnosis of all patients with HCC was confirmed histologically or by the presence of typical hallmarks of HCC using imaging 
techniques of four-phase multi-detector-row CT or dynamic contrast-enhanced MRI (hypervascular lesions in the arterial phase with washout in portal venous or delayed phases). Patients with single HCC and no direct infiltration of the gastrointestinal tract, any intrahepatic metastasis or distant metastasis, and who received $\mathrm{C}$-ion RT as primary treatment were analyzed. Child-Pugh score was calculated for the evaluation of liver function in all patients and the disease stage was determined by CT, MRI, ultrasonography, and other variables in accordance with the Union for International Cancer Control (UICC) classification (seventh edition) (9). The present study complied with the standards of the Declaration of Helsinki and current ethical guidelines and was reviewed and approved by the institutional review board.

Carbon ion radiotherapy. Immobilization devices, consisting of tailor-made fixation cushions and thermoplastic shells, were manufactured for patient fixation. Next, respiratory-gated CT, fourdimensional CT (4D-CT) images for treatment planning, and contrast-enhanced $\mathrm{CT}$ images were acquired. For the precise delineation of the gross tumor volume (GTV), treatment planning $\mathrm{CT}$ images and contrast-enhanced CT images were merged. The clinical target volume (CTV) was defined as a GTV plus $5 \mathrm{~mm}$ in all directions, including microscopic disease progression, and the internal margin (IM) was added as the extent of tumor motion displayed in $4 \mathrm{D}-\mathrm{CT}$ images. The planning target volume (PTV) was defined as a summation of CTV, IM, and setup margin (8). Radiation dose measurements for the target volume and surrounding normal structures were expressed in Gy (relative biologic effectiveness [RBE]), which was defined as the physical dose multiplied by the RBE of carbon ions (10). XiO-N (version 4.47; collaborated product of Elekta AB, Stockholm, Sweden and Mitsubishi Electric, Tokyo, Japan) was used for treatment planning. Prescribed doses were as follows: 52.8 Gy (RBE) or 60.0 Gy (RBE) in four fractions with the planning aim for covering PTV with at least $90 \%$ of the prescribed dose. Dose constraints were as follows: (1) $\mathrm{D}_{1 \mathrm{cc}}<40$ Gy $(\mathrm{RBE})$ administered to the gastrointestinal tract and (2) $\mathrm{V}_{20}<35 \%$ administered to the liver. Dose to the portal vein and bile duct was reduced as much as was possible (8). Figure 1 shows a typical radiation field with dose distribution.

Patients received C-ion RT once daily for four days per week (from Tuesday to Friday). A fiducial gold marker was inserted in the liver for daily patient position matching. Patient positioning with the fiducial marker was confirmed using digital orthogonal X-ray and reference images, which were digitally reconstructed on the basis of CT images for treatment planning (11).

Evaluation during follow-up. Patients were followed up and examined one month after the completion of C-ion RT, and every three months thereafter at our hospital. The follow-up examinations consisted of interview, physical examination, routine blood cell counts, blood chemistry, and abdominal diagnostic imaging, such as four-phase multi-detector-row CT, dynamic contrast-enhanced MRI, or contrast-enhanced ultrasonography. Acute and late toxicities were classified using the National Cancer Institute's Common Terminology Criteria for Adverse Events, version 4.0 (12). Acute toxicity was evaluated as the highest toxicity within three months from the initiation of C-ion RT. Late toxicity was evaluated as the highest toxicity three months after the initiation of the treatments. Local recurrence was defined as tumor regrowth with the enhancement of the contrast effect on CT or MRI or ultrasonography in the irradiated field for the patients treated with C-ion RT.

Assessment of skeletal muscle index and definition of sarcopenia. Cross-sectional areas $\left(\mathrm{cm}^{2}\right)$ of skeletal muscles in the third lumbar vertebrae level were measured by manual outlining on the axial CT images by the radiation oncologist using MIM maestro (version 5.6; MIM Software, Cleveland, OH, USA; Figure 2). Next, crosssectional areas of skeletal muscles were normalized for height $\left(\mathrm{cm}^{2} / \mathrm{m}^{2}\right)$ for calculating skeletal muscle index (SMI). Sarcopenia was defined as SMI less than $43.75 \mathrm{~cm}^{2} / \mathrm{m}^{2}$ for men and less than $41.10 \mathrm{~cm}^{2} / \mathrm{m}^{2}$ for women $(2,13)$.

Data analysis. Prognostic factors were evaluated regarding OS and progression-free survival (PFS) on the basis of the following variables: sarcopenia (presence versus absence), age, sex, SMI, body mass index (BMI; calculated as weight $[\mathrm{kg}] /$ height $\left[\mathrm{m}^{2}\right]$ ), performance status (PS) by Eastern Cooperative Oncology Group classification, serum albumin level, indocyanine green retention test at $15 \mathrm{~min}$ (ICGR15), Child-Pugh class, disease stage according to the UICC classification (seventh edition), and tumor size.

Statistical analysis. Survival was measured from the date of initiation of C-ion RT to the date of death or the most recent follow-up. PFS was measured from the date of initiation of C-ion RT to the date of the first tumor progression. Probabilities regarding OS, local control (LC), and PFS rates were calculated using the Kaplan-Meier method, and the log-rank test was used for comparing the two survival curves for univariate analyses. For determining the implications of potential prognostic factors, univariate and multivariate analyses of OS and PFS were performed using Cox proportional hazards models and the results have been presented as hazard ratios with $95 \%$ confidence interval. Statistical significance in the Cox models was determined using Wald's test. The statistical tests were two-sided, and $p<0.05$ was considered to be statistically significant. Factors with $p<0.1$ in univariate analyses were included in the multivariate analyses. MannWhitney's $U$-test was used for the statistical analysis of differences in patient characteristics noted between the sarcopenia and nonsarcopenia groups. Wilcoxon signed ranks test was used for statistical analyses for the difference in Child-Pugh score between before C-ion $\mathrm{RT}$ and within three months and after three months from the initiation of C-ion RT. All statistical analyses were performed using JMP Pro 12.2.0 software (SAS Institute, Inc., Cary, NC, USA).

\section{Results}

Patient characteristics. A total of 68 patients (22 in the sarcopenia and 46 in the non-sarcopenia groups) were treated with C-ion RT, and all patients completed C-ion RT as per schedule. Patient characteristics of the two groups are summarized in Table I.

Median follow-up for all patients was 33.5 months (range=3.9-83.1 months). Prior treatment for the target region of C-ion RT was surgery in three patients, percutaneous radiofrequency ablation (RFA) in six, RFA with transarterial chemoembolization (TACE) in one, and TACE with surgery in one. No patient received systemic therapy prior to $\mathrm{C}$-ion $\mathrm{RT}$. Dose fractionation schedule was $52.8 \mathrm{~Gy}(\mathrm{RBE}) / 4$ fractions in 37 patients and $60 \mathrm{~Gy}(\mathrm{RBE}) / 4$ fractions in 31 patients. 


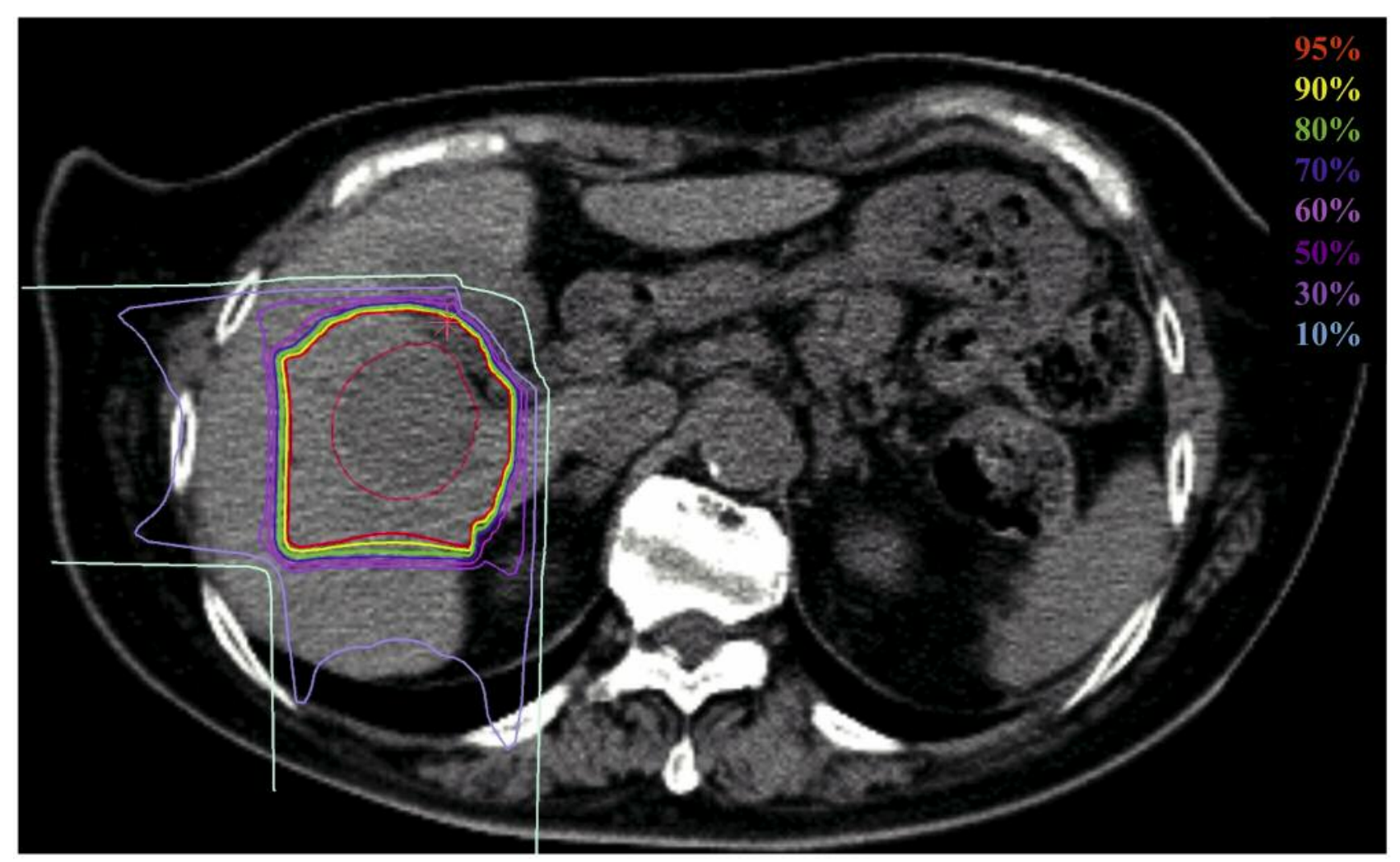

Figure 1. Dose distribution of C-ion RT for HCC. Isodose curves of C-ion RT are superimposed on an axial CT image for the total irradiation plan. The area within the red outline is GTV. Highlighted are), 95\% (red), 90\% (yellow), 80\% (green), 70\% (blue), 60\% (pink), 50\% (purple), 30\% (light purple), and 10\% (light blue) isodose curves (100\% was 52.8 Gy [RBE]).

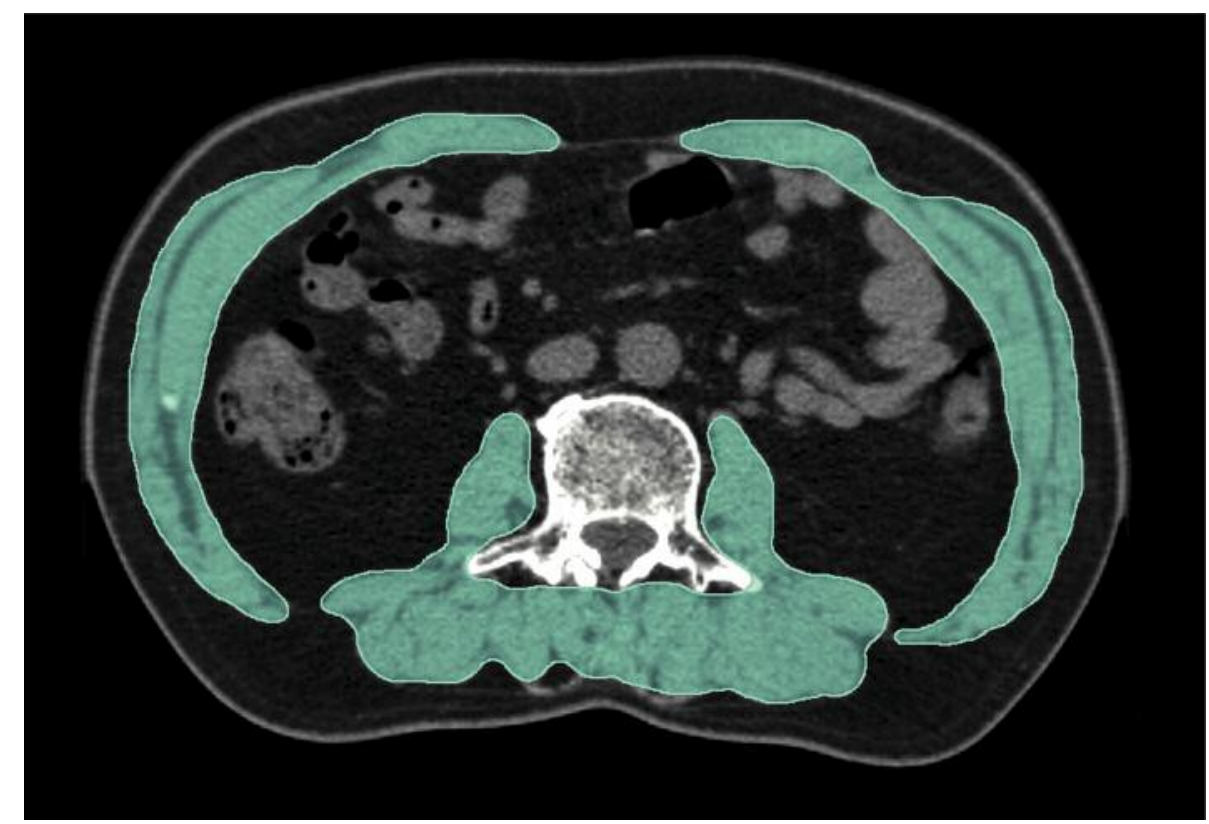

Figure 2. Cross-sectional areas of skeletal muscles in the third lumbar vertebrae level are measured by manual outlining on an axial CT image (highlighted in cyan) for assessing the presence or absence of sarcopenia. 

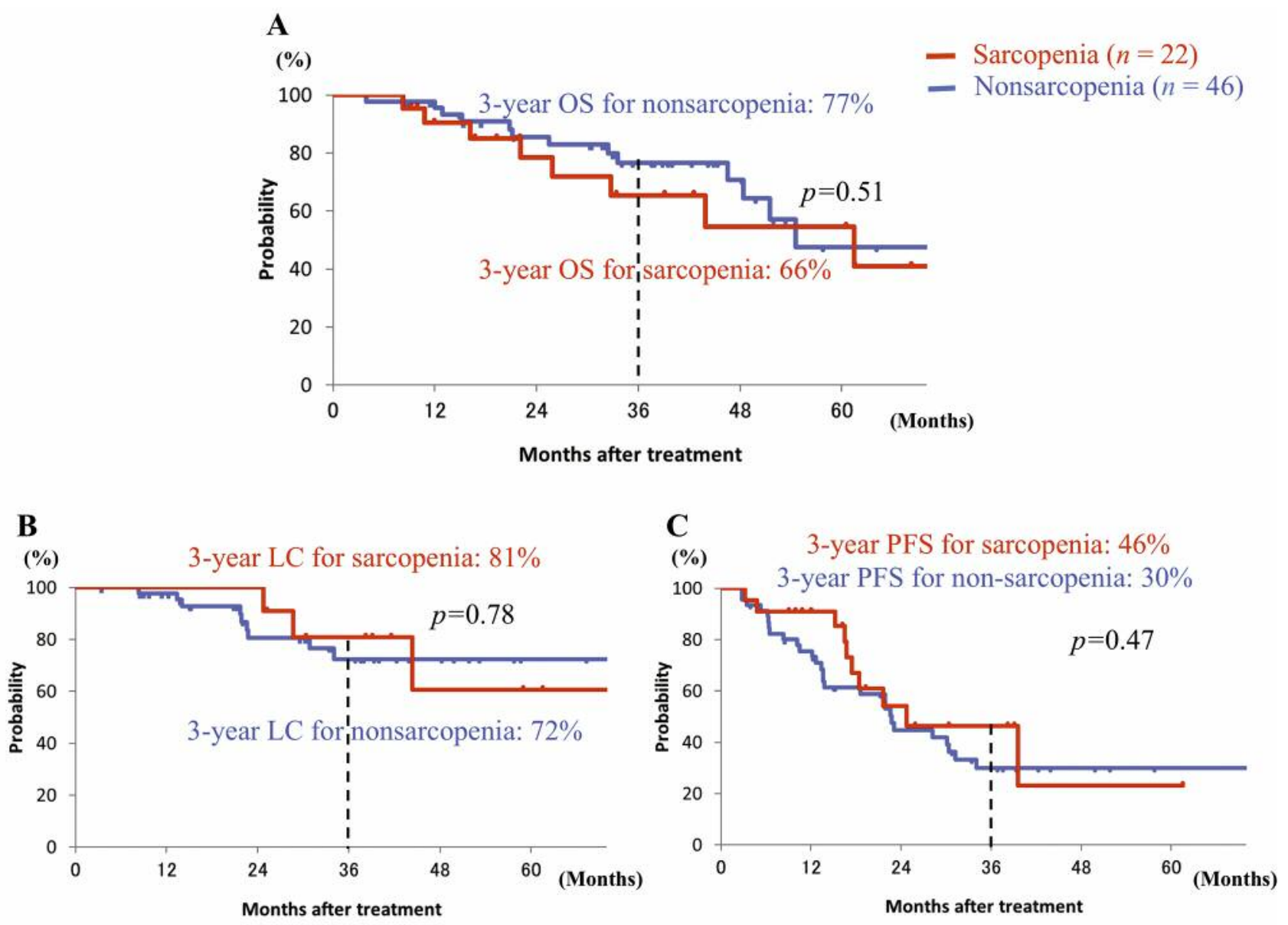

Figure 3. Survival curves of the sarcopenia and non-sarcopenia groups. (A) The overall survival curves of the sarcopenia (red) and non-sarcopenia (blue) groups. (B) The local control curves of the sarcopenia (red) and non-sarcopenia (blue) groups. (C) The PFS curves of the sarcopenia (red) and non-sarcopenia (blue) groups.

OS, LC, and PFS. The OS, LC, and PFS curves of the sarcopenia and non-sarcopenia groups are presented in Figure 3 . The three-year estimated OS, LC, and PFS rates were $66 \%$, $81 \%$, and $46 \%$, respectively, in the sarcopenia group and $77 \%$, $72 \%$, and $30 \%$, respectively, in the non-sarcopenia group ( $p=0.51,0.78,0.47$, respectively; Figure 3 ). At the time of analysis, four patients in the sarcopenia group and eight in the non-sarcopenia group died with HCC as the cause of death, while one and four, respectively, died of intercurrent diseases (pulmonary embolism in the sarcopenia group, and cholangiocarcinoma, renal failure, myocardial infarction, and senility in the non-sarcopenia group).

Toxicity. All observed acute and late toxicities are listed in Table II. Regarding acute toxicities, no patient had Grade 2 or higher radiation dermatitis. One patient in each group experienced Grade 2 elevation of aspartate aminotransferase
(AST). One patient in the sarcopenia group and none in the non-sarcopenia group experienced progression in Child-Pugh class from A to B within three months from the initiation of Cion RT. There was no significant difference noted in the ChildPugh score between before and within three months from initiation of C-ion RT in either group ( $p=0.42$ and $p=0.62$ ).

Regarding late toxicities, two patients in each group experienced Grade 3 encephalopathy. Both patients in the sarcopenia group had progression of HCC and hepatic failure. One of the two patients in the non-sarcopenia group suffered from constipation, and encephalopathy improved with the improvement in constipation, while one experienced a progression of HCC and hepatic failure. Grade 2 ascites occurred in one patient in the sarcopenia group and in two patients in the non-sarcopenia group. Grade 2 elevation of AST occurred in two and one patients, respectively (the latter patient also had elevated alanine aminotransferase 
Table I. Characteristics of patients with sarcopenia and non-sarcopenia.

\begin{tabular}{lccc}
\hline & Sarcopenia $(\mathrm{n}=22)$ & Non-sarcopenia (n=46) & p-Value \\
\hline Age, year, median (range) & $77(57-95)$ & $74(45-90)$ & 0.28 \\
Sex ratio (Male:Female) & $11: 11$ & $30: 16$ & 0.23 \\
SMI, cm ${ }^{2} / \mathrm{m}^{2}$, median (range) & $36.6(28.4-42.9)$ & $48.9(41.3-66.3)$ & $<0.01$ \\
BMI & $19.9(15.7-23.9)$ & $24.7(18.7-32.0)$ & $<0.01$ \\
PS $(0: 1: 2)$ & $12: 10: 0$ & $33: 11: 2$ & 0.22 \\
Albumin & $3.7(2.5-4.2)$ & $3.8(2.8-4.6)$ & 0.17 \\
ICGR15 & $20.2(3.2-44)$ & $19.4(2.3-62.2)$ & 0.84 \\
Child-Pugh class (A:B) & $17: 5$ & $40: 6$ & 0.41 \\
Stage (I:II:III) & $18: 2: 2$ & $39: 5: 2$ & 0.55 \\
Tumor size, mm, median (range) & $30(12-90)$ & $36(9-77)$ & 0.19 \\
\hline
\end{tabular}

BMI: Body mass index; ICGR15: indocyanine green retention test at $15 \mathrm{~min}$; PS: performance status; SMI: skeletal muscle index.

levels). No patient had other late toxicities higher than Grade 2, such as dermatitis, pneumonitis, and rib fracture. Late toxicity progression in Child-Pugh class from A to B occurred in two and one patients. There was no significant difference noted in Child-Pugh score between before and three months after initiation of $\mathrm{C}$-ion RT in either group $(p=0.48$ and $p=0.64)$.

Data analysis. Serum albumin level and Child-Pugh class were identified as prognostic factors for OS after C-ion RT in univariate analysis $(p<0.01$ and $p<0.01$, respectively); however, sarcopenia was not noted to be a prognostic factor $(p=0.51$; Table III). In multivariate analysis, prognostic factors for OS were analyzed on the basis of clinical characteristics of PS, serum albumin level, ICGR15, and Child-Pugh class, and PS and serum albumin level were identified as poor prognostic factors $(p<0.01$ and $p=0.04$, respectively; Table III).

Serum albumin level, ICGR15, and Child-Pugh class were identified as prognostic factors for PFS after C-ion RT in univariate analysis $(p=0.01, p<0.01$, and $p=0.03$, respectively); however, sarcopenia was not noted to be a prognostic factor $(p=0.47$; Table IV). In multivariate analysis, prognostic factors for PFS were analyzed on the basis of clinical characteristics of BMI, serum albumin level, ICGR15, and Child-Pugh class, and Child-Pugh class was identified as a prognostic factor ( $p=0.04$; Table IV).

\section{Discussion}

We analyzed whether the presence or absence of sarcopenia before C-ion RT was a prognostic factor for HCC. The findings of this study indicate that the presence of sarcopenia was not a prognostic factor for OS and PFS in patients with HCC treated with C-ion RT. In other words, C-ion RT showed the same efficacy in patients in both groups.
Table II. Acute and late toxicities by CTCAE, version 4.0.

Acute toxicities in sarcopenia patients $(n=22)$

\begin{tabular}{lrrrrr}
\hline Organs involved & G0 & G1 & G2 & G3 & G4 \\
\hline Dermatitis & 3 & 19 & 0 & 0 & 0 \\
Encephalopathy & 22 & 0 & 0 & 0 & 0 \\
Ascites & 22 & 0 & 0 & 0 & 0 \\
Elevation of AST and/or ALT & 17 & 4 & 1 & 0 & 0
\end{tabular}

Late toxicities sarcopenia patients $(n=22)$

\begin{tabular}{lrrrrr}
\hline Organs involved & G0 & G1 & G2 & G3 & G4 \\
\hline Dermatitis & 11 & 11 & 0 & 0 & 0 \\
Encephalopathy & 20 & 0 & 0 & 2 & 0 \\
Ascites & 17 & 4 & 1 & 0 & 0 \\
Rib bone fracture & 22 & 0 & 0 & 0 & 0 \\
Elevation of AST and/or ALT & 18 & 2 & 2 & 0 & 0
\end{tabular}

Acute toxicities in non-sarcopenia patients $(n=46)$

\begin{tabular}{lrrrrr}
\hline Organs involved & G0 & G1 & G2 & G3 & G4 \\
\hline Dermatitis & 6 & 40 & 0 & 0 & 0 \\
Encephalopathy & 46 & 0 & 0 & 0 & 0 \\
Ascites & 46 & 0 & 0 & 0 & 0 \\
Elevation of AST and/or ALT & 44 & 1 & 1 & 0 & 0 \\
\hline
\end{tabular}

Late toxicities in non-sarcopenia patients $(n=46)$

\begin{tabular}{lrrrrr}
\hline Organs involved & G0 & G1 & G2 & G3 & G4 \\
\hline Dermatitis & 11 & 35 & 0 & 0 & 0 \\
Encephalopathy & 44 & 0 & 0 & 2 & 0 \\
Ascites & 38 & 6 & 2 & 0 & 0 \\
Rib bone fracture & 46 & 0 & 0 & 0 & 0 \\
Elevation of AST and/or ALT & 44 & 1 & 1 & 0 & 0 \\
\hline
\end{tabular}

ALT: Alanine aminotransferase; AST: Aspartate aminotransferase; CTCAE: National Cancer Institute's Common Terminology Criteria for Adverse Events. 
Table III. Univariate and multivariate analyses of clinical factors and overall survival.

\begin{tabular}{|c|c|c|c|c|}
\hline & \multicolumn{2}{|c|}{ Univariate analysis } & \multicolumn{2}{|c|}{ Multivariate analysis } \\
\hline & Hazard ratio & $p$-Value & Hazard ratio & $p$-Value \\
\hline Sarcopenia (presence versus absence) & $1.35(0.53-3.22)$ & 0.51 & & \\
\hline Age & $1.02(0.98-1.08)$ & 0.24 & & \\
\hline Gender & $0.91(0.38-2.20)$ & 0.83 & & \\
\hline SMI & $0.96(0.91-1.01)$ & 0.12 & & \\
\hline BMI & $0.91(0.79-1.04)$ & 0.18 & & \\
\hline PS & $2.18(0.91-5.19)$ & 0.08 & $3.26(1.05-11.39)$ & $<0.01$ \\
\hline Albumin & $0.10(0.04-0.27)$ & $<0.01$ & $0.15(0.04-0.55)$ & 0.04 \\
\hline ICGR 15 & $1.03(0.99-1.05)$ & 0.06 & $1.01(0.99-1.04)$ & 0.33 \\
\hline Child-Pugh class & $3.99(1.50-9.64)$ & $<0.01$ & $2.39(0.59-10.29)$ & 0.22 \\
\hline Stage & $1.45(0.58-2.83)$ & 0.34 & & \\
\hline Tumor size & $1.01(0.98-1.04)$ & 0.36 & & \\
\hline
\end{tabular}

BMI; Body mass index; ICGR15; indocyanine green retention test at 15 min; PS; performance status; SMI; skeletal muscle index.

Table IV. Univariate and multivariate analyses of clinical factors and progression-free survival.

\begin{tabular}{|c|c|c|c|c|}
\hline & \multicolumn{2}{|c|}{ Univariate analysis } & \multicolumn{2}{|c|}{ Multivariate analysis } \\
\hline & Hazard ratio & $p$-Value & Hazard ratio & $p$-Value \\
\hline Sarcopenia (presence versus absence) & $0.77(0.35-1.53)$ & 0.47 & & \\
\hline Age & $1.00(0.97-1.04)$ & 0.95 & & \\
\hline Gender & $1.05(0.56-2.07)$ & 0.87 & & \\
\hline SMI & $1.01(0.97-1.04)$ & 0.74 & & \\
\hline BMI & $1.08(0.99-1.19)$ & 0.09 & $1.05(0.95-1.16)$ & 0.37 \\
\hline PS & $1.11(0.54-2.15)$ & 0.77 & & \\
\hline Albumin & $0.39(0.19-0.82)$ & 0.01 & $0.69(0.29-1.64)$ & 0.40 \\
\hline ICGR 15 & $1.02(1.00-1.04)$ & 0.04 & $1.01(0.99-1.03)$ & 0.41 \\
\hline Child-Pugh class & $4.42(1.78-10.11)$ & $<0.01$ & $2.86(1.01-7.89)$ & 0.04 \\
\hline Stage & $1.23(0.65-2.02)$ & 0.47 & & \\
\hline Tumor size & $0.98(0.97-1.01)$ & 0.19 & & \\
\hline
\end{tabular}

BMI: Body mass index; ICGR15: indocyanine green retention test at $15 \mathrm{~min}$; PS: performance status; SMI: skeletal muscle index.

Sarcopenia has been reported as a prognostic factor for HCC patients treated with hepatic resection with curative intent as the initial treatment $(2,4,14)$. These results were obtained from patient characteristics in which median age was 62-69 years and the frequency of Child-Pugh class A was $94-100 \%$. Regarding patient characteristics, if the patient's condition in our study was better than that in surgical studies, it may indicate good prognosis in patients with sarcopenia. However, patient characteristics in our study, such as age or Child-Pugh class, were higher compared with those in the surgical studies. In this study, median age was 76 years and frequency of Child-Pugh class A was $84 \%$. In general, higher age or Child-Pugh class was strongly correlated with poor prognosis (15). Thus, patient characteristics in our study did not contribute to good results in patients with sarcopenia.

The invasiveness of cancer treatment was considered a possible reason for the difference noted between our study and surgical studies in the prognosis of sarcopenia. First, the frequency of severe toxicities may have affected the results. Regarding acute toxicities, no patient had Grade 3 or higher acute toxicities in our study. In contrast, $18 \%$ of the patients in a surgical study had Grade 3 or higher acute toxicities (4). Second, fasting duration during treatment may have been associated with the results showing the same efficacy in the sarcopenia as in the non-sarcopenia groups. Patients who underwent surgery had 2.8 days of postoperative fasting (16). In contrast, patients who underwent $\mathrm{C}$-ion $\mathrm{RT}$ had no fasting 
duration, and there was no deterioration of nutritional condition. Longer fasting duration was thus considered to worsen nutrition in patients with sarcopenia and to affect prognosis after surgery. Third, liver functional reserve after cancer treatment may have affected the results of this study. Liver functional reserve is reportedly one of the crucial factors determining survival (15). C-ion RT has an excellent dose distribution property and enables the delivery of a high dose to tumors while minimizing damage to normal tissue (7) so that normal liver suffered less damage. In this study, only two patients $(9 \%)$ in the sarcopenia group experienced the progression of Child-Pugh class from A to B as late toxicity. This result suggested that $\mathrm{C}$-ion RT could be a safe treatment for patients with sarcopenia with similar survival as for patients with non-sarcopenia.

Previous report indicate that the improvement of malnutrition by the intervention of a nutrition support team and early physical therapy intervention aimed at improving skeletal muscle mass are considered to improve the survival of patients with sarcopenia (17-19). There are no fasting duration and postoperative activity restrictions in C-ion RT; thus, nutrition and physical therapy interventions could be initiated from the commencement of treatment without any interruption during C-ion RT.

Numerous local treatment options are present for treating HCC. We compared survival in this study to that of other studies using other cancer treatments, such as surgery, TACE, and RFA. The clinical outcomes of hepatectomy $(11,20,21)$ TACE (22), and RFA $(20,22,23)$ have been previously reported, with three-year OS rates of $82 \%, 57 \%$, and $81 \%$, respectively. The above-mentioned results of other modalities were obtained from patients regardless of the presence or absence of sarcopenia. In this study, the three-year OS rates were $66 \%$ and $77 \%$ in the sarcopenia and non-sarcopenia groups, respectively. Even though the number of patients in this study was small, our results supported the continued evaluation of efficacy compared to surgery, TACE, and RFA.

This study had a few limitations. First, this is a single institutional analysis with a limited number of patients and insufficient statistical power. Second, the retrospective nature makes this study vulnerable to potential biases. Third, we analyzed only Japanese patients, so the results of this study may not be directly applicable to various ethnic populations. However, to our knowledge, this is the first study to demonstrate that sarcopenia is not a prognostic factor for clinical outcomes of patients with HCC treated with C-ion RT.

In conclusion, sarcopenia was not a prognostic factor for patients with HCC treated with C-ion RT. C-ion RT for patients with $\mathrm{HCC}$ and sarcopenia was effective with minimal toxicities without worsening survival. This result suggested that $\mathrm{C}$-ion RT may become an alternative treatment option for patients with HCC and sarcopenia for whom other treatment, such as surgery, was not a viable choice.

\section{Acknowledgements}

The Authors wish to thank all patients who were involved in this study and our colleagues at the Department of Radiation Oncology, Gunma University Graduate School of Medicine. This work was financially supported by Grants-in-Aid from the Ministry of Education, Culture, Sports, Science, and Technology of Japan for programs for Leading Graduate Schools, Cultivating Global Leaders in Heavy Ion Therapeutics and Engineering.

\section{References}

1 Fujiwara N, Nakagawa H, Kudo Y, Tateishi R, Taguri M, Watadani T, Nakagomi R, Kondo M, Nakatsuka T, Minami T, Sato M, Uchino K, Enooku K, Kondo Y, Asaoka Y, Tanaka Y, Ohtomo K, Shiina S and Koike K: Sarcopenia, intramuscular fat deposition, and visceral adiposity independently predict the outcomes of hepatocellular carcinoma. J Hepatol 63: 131-140, 2015.

2 Harimoto N, Shirabe K, Yamashita YI, Ikegami T, Yoshizumi T, Soejima Y, Ikeda T, Maehara Y, Nishie A and Yamanaka T: Sarcopenia as a predictor of prognosis in patients following hepatectomy for hepatocellular carcinoma. Br J Surg 100: 1523$1530,2013$.

3 Hodari A, Hammoud ZT, Borgi JF, Tsiouris A and Rubinfeld IS: Assessment of morbidity and mortality after esophagectomy using a modified frailty index. Ann Thorac Surg 96: 1240-1245, 2013.

4 Voron T, Tselikas L, Pietrasz D, Pigneur F, Laurent A, Compagnon P, Salloum C, Luciani A, and Azoulay D: Sarcopenia impacts on short- and long-term results of hepatectomy for hepatocellular carcinoma. Ann Surg 261: 1173-1183, 2015.

5 Prado CM, Lieffers JR, McCargar LJ, Reiman T, Sawyer MB, Martin L and Baracos VE: Prevalence and clinical implications of sarcopenic obesity in patients with solid tumours of the respiratory and gastrointestinal tracts: a population-based study. Lancet Oncol 9: 629-635, 2008.

6 Ferlay J, Shin HR, Bray F, Forman D, Mathers C and Parkin DM: Estimates of worldwide burden of cancer in 2008: GLOBOCAN 2008. Int J Cancer 127: 2893-2917, 2010.

7 Abe T, Saitoh J, Kobayashi D, Shibuya K, Koyama Y, Shimada H, Shirai K, Ohno T and Nakano T: Dosimetric comparison of carbon ion radiotherapy and stereotactic body radiotherapy with photon beams for the treatment of hepatocellular carcinoma. Radiation Oncol (London, England) 10: 187, 2015.

8 Shiba S, Abe T, Shibuya K, Katoh H, Koyama Y, Shimada H, Kakizaki S, Shirabe K, Kuwano H, Ohno T and Nakano T: Carbon ion radiotherapy for 80 years or older patients with hepatocellular carcinoma. BMC Cancer 17: 721, 2017.

9 Sobin LH, Gospodarowicz MK and Wittekind C: TNM classification of malignant tumours: John Wiley \& Sons, 2011.

10 Inaniwa T, Kanematsu N, Matsufuji N, Kanai T, Shirai T, Noda K, Tsuji H, Kamada T and Tsujii H: Reformulation of a clinicaldose system for carbon-ion radiotherapy treatment planning at the National Institute of Radiological Sciences, Japan. Phys Med Biol 60: 3271-3286, 2015.

11 Abe S, Kubota Y, Shibuya K, Koyama Y, Abe T, Ohno T and Nakano T: Fiducial marker matching versus vertebral body matching: Dosimetric impact of patient positioning in carbon ion radiotherapy for primary hepatic cancer. Phys Med 33: 114-120, 2017. 
12 U.S. Department of Health and Human Services: Common terminology criteria for adverse events (CTCAE) version 4.0. National Institutes of Health, National Cancer Institute 42009.

13 Parkin E, Plumb AA, O'Reilly D and Renehan AG: Body composition and outcome in patients undergoing resection of colorectal liver metastases (Br J Surg 2012; 99: 550-557). Br J Surg 99: 1021-1022, 2012.

14 Takagi K, Yagi T, Yoshida R, Shinoura S, Umeda Y, Nobuoka D, Kuise T, Watanabe N and Fujiwara T: Sarcopenia and American Society of Anesthesiologists physical status in the assessment of outcomes of hepatocellular carcinoma patients undergoing hepatectomy. Acta Med Okayama 70: 363-370, 2016.

15 Kudo M, Chung H and Osaki Y: Prognostic staging system for hepatocellular carcinoma (CLIP score): its value and limitations, and a proposal for a new staging system, the Japan Integrated Staging Score (JIS score). J Gastroenterol 38: 207-215, 2003.

16 Yoon SY, Kim KH, Jung DH, Yu A and Lee SG: Oncological and surgical results of laparoscopic versus open liver resection for HCC less than $5 \mathrm{~cm}$ : case-matched analysis. Surg Endosc 29: 2628-2634, 2015.

17 Anand AC: Nutrition and muscle in cirrhosis. J Clin Exp Hepatol 7: 340-357, 2017.

18 Hayaishi S, Chung H, Kudo M, Ishikawa E, Takita M, Ueda T, Kitai S, Inoue T, Yada N, Hagiwara S, Minami Y and Ueshima K: Oral branched-chain amino acid granules reduce the incidence of hepatocellular carcinoma and improve event-free survival in patients with liver cirrhosis. Dig Dis 29: 326-332, 2011.

19 Koya S, Kawaguchi T, Hashida R, Goto E, Matsuse H, Saito H, Hirota K, Taira R, Matsushita Y, Imanaga M, Nagamatsu A, Shirono T, Shimose S, Iwamoto H, Niizeki T, Kuromatsu R, Miura H, Shiba N and Torimura T: Effects of in-hospital exercise on liver function, physical ability, and muscle mass during treatment of hepatoma in patients with chronic liver disease. Hepatol Res 47: E22-e34, 2017.
20 Liu PH, Hsu CY, Lee YH, Hsia CY, Huang YH, Su CW, Chiou YY, Lin HC and Huo TI: Uncompromised treatment efficacy in elderly patients with hepatocellular carcinoma: A propensity score analysis: erratum. Medicine 94: 1, 2015.

21 Huang J, Li BK, Chen GH, Li JQ, Zhang YQ, Li GH and Yuan YF: Long-term outcomes and prognostic factors of elderly patients with hepatocellular carcinoma undergoing hepatectomy. J Gastrointest Surg 13: 1627-1635, 2009.

22 Takahashi H, Mizuta T, Kawazoe S, Eguchi Y, Kawaguchi Y, Otuka T, Oeda S, Ario K, Iwane S, Akiyama T, Ozaki I and Fujimoto K: Efficacy and safety of radiofrequency ablation for elderly hepatocellular carcinoma patients. Hepatol Res 40: 9971005, 2010.

23 Wu J, Lin S, Wu W, Yan K, Dai Q and Chen M: [Clinical application of ultrasound-guided radiofrequency ablation for primary hepatocellular carcinoma near the liver surface]. Zhonghua Zhong Liu Za Zhi 37: 933-937, 2015.
Received April 5, 2018

Revised April 24, 2018

Accepted April 25, 2018 